\title{
Analysis of X-ray Diffraction Line Broadening using a Non-ideal Standard Specimen
}

\author{
Th. H. de Keijser and E. J. Mittemeijer \\ Laboratory of Metallurgy, Delft University of Technology, Rotterdamseweg 137, Delft, \\ The Netherlands
}

Z. Naturforsch. 33 a, 316-320 (1978) ; received December 18, 1977

\begin{abstract}
In practice a standard specimen used for the elimination of instrumental broadening, by deconvolution, is never ideal. The instrumental line profile always contains some additional broadening due to finite particle size and distortion present in the standard specimen. Further it is sometimes unavoidable to use a standard specimen of a material different from the material to be analyzed, leading to reflections at incorrect $\sin \theta$-values (different spectral broadening). Firstly the effect of these deficiencies on the Fourier coefficients of the pure line profile is analyzed. Secondly the consequences for size-strain analysis are considered.

A correction [Eq. (3.4)] for the difference in spectral broadening between the exact and the approximate instrumental line profile is given. To avoid serious errors, this correction should be performed before particle size and strain are determined according to the so-called WarrenAverbach analysis. Accurate relative determinations of size and strain are possible even in the case that the line profile under investigation and the line profile of the reference specimen are at different $\sin \theta$-values. It is suggested that standard specimens which cause noticeable structural broadening can be used profitably.
\end{abstract}

\section{Introduction}

The measured line profile $h$ is the convolution of the structurally broadened profile $f$ with the instrumental profile $g$. From the $h$ and $g$ profiles, the pure profile $f$ can be obtained by the deconvolution method of Stokes [1]. Because of unavoidable alignment errors [2] the instrumental profile has to be determined experimentally from a so-called standard specimen. With the origin for the $g$ - and $h$-profiles chosen at the same value of $\sin \theta$ and $F$, $G$ and $H$ being the Fourier coefficients of the $f-, g$ and $h$-profiles respectively, it follows

$$
F(n)=\frac{A}{a} \frac{H(n)}{G(n)}=G(0) \frac{H(n)}{G(n)},
$$

where $n$ is the harmonic number, $A$ is the integrated intensity and $a$ the period taken for $g$ and $h$ on a $\sin \theta$-scale. The Fourier coefficients $F(n)$ are the basis for the size-strain analysis originally developed by Warren and Averbach [3, 4].

Different sources of errors occurring in the Fourier coefficients have been considered in the literature. The errors caused by sampling and truncation of the profile have been treated by Brigham [5] and Young, Gerdes and Wilson [6], while the latter authors also calculated the errors in the Fourier coefficients caused by an inaccurate choice of background. Wilson [7-9] considered the influence of counting statistics.

Reprint requests to Dr. Ir. Th. H. de Keijser, Laboratory of Metallurgy, Delft University of Technology, Rotterdamseweg 137, Delft, The Netherlands.
So far little attention has been paid to the error caused by an approximate instrumental line profile. In principle it is impossible to measure the exact $g$-profile. At best the "instrumental" profile is recorded from a standard specimen of the same composition that is treated in the same way, as far as possible, as the specimen used for the $h$-profile. In many cases an "instrumental" profile has to be taken from a standard specimen different in composition [10] or even made of an other material [11]. This leads to instrumental line profiles measured at incorrect $\theta$-values. Furthermore some structural broadening may be present in the instrumental line profile [12].

A solution to the problem of the incorrect $\theta$-value may be obtained by applying the profile fitting method [13]. In this method an instrumental line profile is approximated by a small number of Cauchy-functions. Several instrumental line profiles taken over a large range of $\boldsymbol{\theta}$-values are measured. The parameters for the Cauchy-functions are obtained by fitting. Normally an instrumental line profile is needed at an unmatched $\theta$-value. The parameters necessary for the evaluation in terms of Cauchy-functions of that profile are obtained by interpolation. However, in case of size-strain analysis on the basis of the method developed by Warren and Averbach, which is adopted in our paper, the Fourier coefficients of the pure profile are required. Therefore a direct description in terms of Fourier coefficients is preferable. For the determination of Fourier coefficients of instrumental 
profiles at unmatched $\theta$-values an analogous interpolation procedure can be followed as for the determination of the parameters of the Cauchyfunctions.

Although the above mentioned interpolation procedures are possible methods for size-strain analysis, it should be recognized that these procedures are laborious and still don't give exact results. Moreover any change in the experimental conditions (e. g. slit width, X-ray spectrum [14], absorption in the specimen) obliges to measure again the instrumental profiles. This may be the reason that, to our knowledge, no Warren-Averbach analysis on the basis of the profile fitting method has been reported in the literature.

In this paper an alternative, analytical and quick method is proposed to correct for instrumental profiles measured at incorrect $\theta$-values. The method is based on an analytical description of the $\mathrm{K} \alpha$ doublet. An experimental justification for this description is given. Further the effect of the structural broadening present in the instrumental line profile is analyzed.

\section{The Effect of an Approximate Instrumental Line Profile on the Fourier coefficients of the Pure Profile}

In the approximate instrumental line profile two types of deviations are distinguished:

(i) the physical profile $f_{0}$ of the standard specimen is not a delta function, but it is (slightly) broadened by strain and finite particle size;

(ii) the instrumental profile $g_{\mathrm{m}}$ is measured at a position on the $\sin \theta$-scale different from that of the desired exact instrumental profile $g$.

In general the approximate instrumental profile is the convolution of $f_{0}$ and $g_{\mathrm{m}}$. According to Eq. (1.1) the Fourier coefficients of the approximate instrumental profile are equal to $F_{0}(n) G_{\mathrm{m}_{\mathrm{L}}}(n)$, where $\mathrm{F}_{0}(n)$ and $G_{\mathrm{m}}(n)$ are the Fourier coefficients of the $f_{0}$ and $g_{\mathrm{m}}$ profiles, respectively, and $F_{0}(n)$ is normalized such that $F_{0}(0)=1$. In practice then the approximate Fourier coefficients for the pure profile are obtained from

$$
F_{\mathrm{m}}(n)=G_{\mathrm{m}}(0) \frac{H(n)}{F_{0}(n) G_{\mathrm{m}}(n)}
$$

Taking $G(0)=G_{\mathrm{m}}(0)=1$ from Eqs. (1.1) and
(2.1) it follows

$$
F_{\mathrm{m}}(n)=\frac{G(n)}{G_{\mathrm{m}}(n)} \frac{F(n)}{F_{0}(n)} .
$$

In order to find the relation between $F_{\mathrm{m}}(n)$ and $F(n), G(n)$ must be related to $G_{\mathrm{m}}(n)$, which means that $G(n)$ must be known as a function of $\sin \theta$.

Recently we proposed the following model for the instrumental profile [15]. The instrumental line profile $g(x)$, with $x$ defined on a $\sin \theta$-scale [16], is taken as the sum of the $\alpha_{1}$ and $\alpha_{2}$ components. The shape and position of the components is related to an instrumental profile $g_{\mathrm{c}}(x)$ intermediate between that of the $\alpha_{1}$ and $\alpha_{2}$ components. $g_{\mathrm{c}}(x)$ is considered as the convolution of the spectral profile $g_{\mathrm{c}}{ }^{\mathrm{s}}(x)$ of the intermediate wavelength distribution and the profile $g_{\mathrm{c}}{ }^{\mathrm{i}}(x)$ due to the broadening caused by the instrumental conditions. For $g_{\mathrm{c}}^{\mathrm{s}}(x)$ the generally accepted Cauchy wavelength distribution is adopted $[2,17]$. This leads to

$$
\begin{aligned}
G(n)= & G_{\mathrm{c}}^{\mathrm{i}}(n) \frac{1}{1+R} \\
& \cdot[\exp \{-i n R \alpha\}+R \exp \{i n \alpha\}] \\
& \quad \exp \{-\pi n w / 2 d a\}
\end{aligned}
$$

where $G_{\mathrm{c}}{ }^{\mathrm{i}}(n)$ is the Fourier coefficient of $g_{\mathrm{c}}{ }^{\mathrm{i}}(x)$ normalized such that $G_{\mathrm{c}} \mathrm{i}(0)=1$;

$$
\alpha=\pi\left(\left\langle\lambda_{2}\right\rangle-\left\langle\lambda_{1}\right\rangle\right) / d(1+R) a
$$

where $\left\langle\lambda_{1}\right\rangle$ and $\left\langle\lambda_{2}\right\rangle$ are the centroids of the $\alpha_{1}$ and $\alpha_{2}$ wavelength distributions respectively, $d$ is the spacing of the reflecting planes and $a$ is the period on a $\sin \theta$-scale used for the calculation of the Fourier coefficients; $w$ is the width at half height of the intermediate wavelength distribution $g_{\mathrm{c}}^{\mathrm{s}}(\lambda)$. The centroid of $g_{\mathrm{c}}^{\mathrm{s}}(\lambda)$ is given by $\left\langle\lambda_{\mathrm{c}}\right\rangle=\left(\left\langle\lambda_{1}\right\rangle+\right.$ $\left.R\left\langle\lambda_{2}\right\rangle\right) /(1+R)$, where $R=I \alpha_{2}(\max ) / I \alpha_{1}(\max )$.

In this paper the validity of Eq. (2.3) is experimentally verified. It may be assumed that the instrumental broadening of a high angle reflection is entirely due to the X-ray spectrum used [13]. Then $g_{\mathrm{c}}{ }^{\mathrm{i}}(x)$ is a delta-function and $G_{\mathrm{c}}{ }^{\mathrm{i}}(n)=1$ [the centroid of $g_{\mathrm{c}}{ }^{\mathrm{i}}(x)$ chosen at $\left.x=0\right]$. From Fig. 1 it is concluded that Eq. (2.3) gives a proper description of the spectral broadening, in particular for the smaller values of $n$, which are of interest in sizestrain analysis.

Applying Eq. (2.3), an expression for $G_{\mathrm{m}}(n)$ is obtained by replacing $G(n)$ by $G_{\mathrm{m}}(n), G_{\mathrm{c}}{ }^{\mathrm{i}}(n)$ by $G_{\mathrm{c}, \mathrm{m}}^{\mathrm{i}}(n)$ and $d$ (also in $\alpha$ ) by $d_{\mathrm{m}}$, thes pacing of 


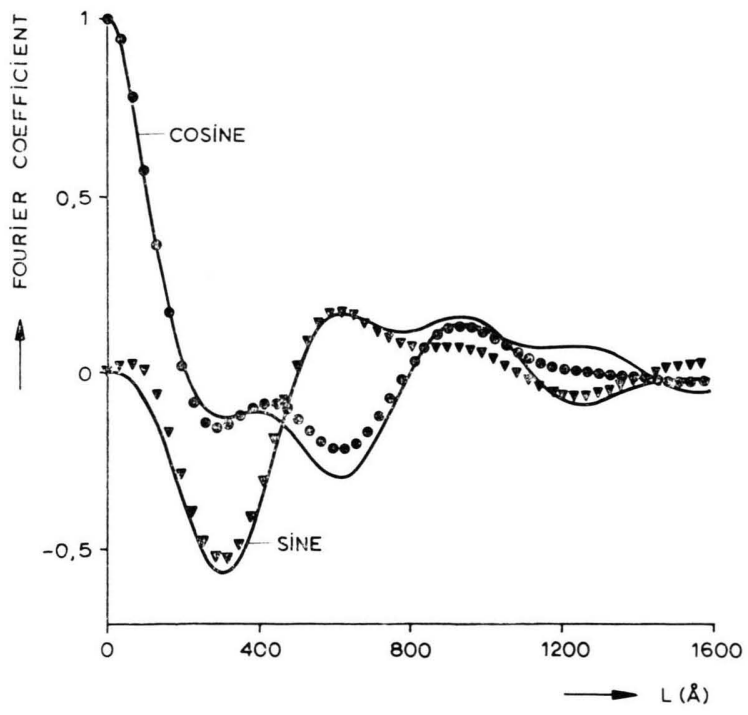

Fig. 1. Calculated and experimental Fourier coefficients as a function of the distance $L=n\left\langle\lambda_{\mathrm{c}}\right\rangle / 2 a$, perpendicular to the reflecting planes, for the (444) reflection of silicon recorded with the $\operatorname{CuK} \alpha$ doublet. Full lines: the cosine and sine coefficients calculated from Eq. (2.3) with $G_{\mathrm{c}^{\mathrm{i}}}(n)=1$ and $w=6.5 \times 10^{-4} \AA$ [17]. - The symbols $\bigcirc$ and $\boldsymbol{\nabla}$ denote the cosine and sine coefficients respectively of the experimental profile. Divergence and receiving slits were $0.5^{\circ}$ and $0.05 \mathrm{~mm}$ respectively. Soller slits with $4^{\circ}$ divergence were used. A standard silicon powder sample [Nat. Bur. of Standards SRM 640] was applied.

the reflecting planes in the approximate standard specimen.

If $d_{\mathrm{m}}$ is chosen sufficiently close to $d$ it can be shown [15] for the broadening due to the instrumental factors that for not too large $n$ and not too small $\theta$

$$
G_{\mathrm{c}}^{\mathrm{i}}(n) / G_{\mathrm{c}, \mathrm{m}}^{\mathrm{i}}(n)=1
$$

where the centroids of the $g$-profile and of the $g_{\mathrm{m}}$ profile (which are at different $\sin \theta$ values) are identified with the origin of the $x$-axis. With Eqs. $(2.2),(2.3)$ and $(2.4)$ the Fourier coefficient $F(n)$ of the pure line profile can be calculated.

In the following the consequences for size-strain analysis will be investigated.

\section{The Effect of an Approximate Instrumental Line Profile in Size-strain Analysis}

Following the Warren-Averbach analysis $[3,4]$ the normalized Fourier coefficients $F(n) / F(0)$ of a structurally broadened profile can be written as the product of a "size" Fourier coefficient $A^{\mathrm{S}}(n)$ which is real and independent of the order $l$ of the re- flection and a "distortion" Fourier coefficient $F^{\mathrm{D}}(n, l)=A^{\mathrm{D}}(n, l)+i B^{\mathrm{D}}(n, l)$.

It should be remarked that in the Warren-Averbach analysis the Fourier series evaluation of the pure $(00 l)$ line profile is performed over the range $l-\frac{1}{2}$ to $l+\frac{1}{2}$ of the variable $h_{3}=2 a_{3} \sin \theta /\left\langle\lambda_{\mathrm{c}}\right\rangle$, where $a_{3}$ is the spacing of the (001) planes [18]. In this paper the Fourier analysis is performed on a $\sin \theta$-scale over a range $a=\left\langle\lambda_{\mathrm{c}}\right\rangle / 2 a_{3}$.

The following expression was given for $A^{\mathrm{D}}(n, l)$

$$
\ln A^{\mathrm{D}}(n, l)=-2 \pi^{2} n^{2} l^{2}\left\langle e^{2}(n)\right\rangle
$$

where $\left\langle e^{2}(n)\right\rangle$ is the mean square strain in the specimen. If (at least) two orders of a reflection are available $A^{\mathrm{S}}(n)$ and $\left\langle e^{2}(n)\right\rangle$ can be found separately from a plot of $\ln A(n, l)$ versus $l^{2}$. Recently Delhez and Mittemeijer [19] have shown that plotting $A(n, l)$ versus $l^{2}$ is more accurate. The relations between measured and true values for particle size and mean square strain and the conclusions presented in this paper are the same for both methods of separation.

By power series expansion the term in brackets of Eq. (2.3) can be approximated:

$$
\begin{array}{r}
{[\exp \{-i n R \alpha\}+R \exp \{i n \alpha\}]} \\
\cong(1+R)\left[1-n^{2} R \alpha^{2} / 2\right] .
\end{array}
$$

Thus, within the approximation used, this factor is real.

Assuming the distortion sine coefficients $B_{0}{ }^{\mathrm{D}}(n, l)$ of the standard specimen to be zero (which is exact for symmetrical distortion broadening) and using the relations $d=a_{3} / l$ and $2 a a_{3}=\left\langle\lambda_{\mathrm{c}}\right\rangle$ one obtains from Eqs. (2.2), (2.3) and (2.4) with Eq. (3.2)

$$
\begin{aligned}
A_{\mathrm{m}}(n, l)= & \frac{A^{\mathrm{S}}(n)}{A_{0}^{\mathrm{S}}(n)} \frac{A^{\mathrm{D}}(n, l)}{A_{0} \mathrm{D}(n, l)} \frac{1-n^{2} l^{2} \beta}{1-n^{2} l^{2} r^{2} \beta} \\
& \cdot \exp \left\{\frac{\pi n l w(1-r)}{\left\langle\lambda_{\mathrm{c}}\right\rangle}\right\}
\end{aligned}
$$

where $\beta=2 \pi^{2}\left(\left\langle\lambda_{2}\right\rangle-\left\langle\lambda_{1}\right\rangle\right)^{2} R /(1+R)^{2}\left\langle\lambda_{c}\right\rangle^{2}$ and $r=d / d_{\mathrm{m}}$. From Eq. (3.3) $\mathrm{i}$ is clear that a plot of $\ln A_{\mathrm{m}}(n, l)$ versus $l^{2}$ does not result in a determination of $A^{\mathrm{S}}(n) / A_{0} \mathrm{~S}(n)$ and $A^{\mathrm{D}}(n, l) / A_{0} \mathrm{D}(n, l)$ separately, because of the factor $\exp \{-\pi n l w(1-r) /$ $\left.\left\langle\lambda_{\mathrm{c}}\right\rangle\right\}$. Since all quantities in the last two factors of Eq. (3.3) are known a corrected Fourier coefficient is defined

$$
\begin{aligned}
A_{\mathrm{m}}^{\mathrm{corr}}(n, l)= & A_{\mathrm{m}}(n, l) \frac{1-n^{2} l^{2} r^{2} \beta}{1-n^{2} l^{2} \beta} \\
& \cdot \exp \left\{\frac{\pi n l w(1-r)}{\left\langle\lambda_{\mathrm{c}}\right\rangle}\right\} .
\end{aligned}
$$


Using Eq. (3.1), from Eqs. (3.3) and (3.4) it is obtained

$$
\begin{aligned}
\ln A_{\mathrm{m}}^{\mathrm{corr}}(n, l)= & \ln \frac{A^{\mathrm{S}}(n)}{A_{0}^{\mathrm{S}}(n)}-2 \pi^{2} n^{2} l^{2} \\
& \cdot\left[\left\langle e^{2}(n)\right\rangle-\left\langle e_{0}^{2}(n)\right\rangle\right]
\end{aligned}
$$

where $\left\langle e_{0}^{2}(n)\right\rangle$ is the mean square strain in the standard specimen. Following Delhez and Mittemeijer [19] the expression (3.5) should be replaced by

$$
\begin{aligned}
A_{\mathrm{m}}^{\text {corr }}(n, l)= & \frac{A^{\mathrm{S}}(n)}{A_{0}{ }^{\mathrm{S}}(n)}-\frac{A^{\mathrm{S}}(n)}{A_{0}{ }^{\mathrm{S}}(n)} 2 \pi^{2} n^{2} l^{2} \\
& \cdot\left[\left\langle e^{2}(n)\right\rangle-\left\langle e_{0}{ }^{2}(n)\right\rangle\right] .
\end{aligned}
$$

A plot of $\ln A_{\mathrm{m}}^{\text {corr }}(n, l)$ versus $l^{2}$ [Eq. (3.5)] and a plot of $A_{\mathrm{m}}^{\text {corr }}(n, l)$ versus $l^{2}[\mathrm{Eq}$. (3.6)] both yield

$$
A_{\mathrm{m}}{ }^{\mathrm{S}}(n)=A^{\mathrm{S}}(n) / A_{0}{ }^{\mathrm{S}}(n)
$$

and

$$
\left\langle e_{\mathrm{m}}^{2}(n)\right\rangle=\left\langle e^{2}(n)\right\rangle-\left\langle e_{0}^{2}(n)\right\rangle .
$$

The mean particle size and the particle size distribution may be obtained from Equation (3.7) [3]. $N_{3}$, the mean particle size expressed in units $a_{3}$, is obtained from $\mathrm{d} A^{\mathrm{S}}(n) / \mathrm{d} n$ (for $\left.n \rightarrow 0\right)=-1 / N_{3}$. Then the relation between the measured particle size $N_{3, \mathrm{~m}}$, the true particle size $N_{3}$ and the particle size $N_{3,0}$ of the standard specimen follows from Eq. (3.7)

$$
\left(N_{3, \mathrm{~m}}\right)^{-1}=\left(N_{3}\right)^{-1}-\left(N_{3,0}\right)^{-1} .
$$

In an X-ray diffraction study of deformation in Ag - Si alloys by Zemitis, Kidron and Cohen [21] formulae related to Eq. (3.8) and Eq. (3.9) were used. These equations were applied for relative determinations. From the treatment given in this paper it may be concluded that accurate relative determinations using Eqs. (3.8) and (3.9) are possible even in the case that the line profile under investigation and the line profile of the reference specimen are at different $\sin \theta$-values, provided the correction implied by Eq. (3.4) has been performed. Furthermore from these relations the accuracy of size-strain analysis can be estimated.

[1] A. R. Stokes, Proc. Phys. Soc. London 61, 382 (1948).

[2] H. P. Klug and L. E. Alexander, X-ray Diffraction Procedures, John Wiley and Sons, New York 1974, pp. $295 \mathrm{ff}$.

[3] B. E. Warren and B. L. Averbach, J. Appl. Phys. 21, 595 (1950).

[4] B. E. Warren and B. L. Averbach, J. Appl. Phys. 23, 497 (1952).
If the corrections implied by Eq. (3.4) and Eqs. (3.8) and (3.9) are ignored errors in the values calculated for particle size and mean square strain will occur. Especially the accuracy of the mean square strain determined is heavily affected. A preliminary calculation already showed errors in the order of $50 \%$ to be possible [22]. In that calculation only the difference between the widths of the spectral components of ideal and approximate instrumental profile was taken into account. In this paper also the influence of the difference in doublet separation between ideal and approximate instrumental profile and the influence of structural broadening by the standard specimen is considered. From the complete equations it follows that the error mentioned can be even larger.

From Eq. (3.3) it follows

$$
\left.\frac{\mathrm{d} A_{\mathrm{m}}(n, l)}{d n}\right|_{n \rightarrow 0}=-\frac{1}{N_{3, \mathrm{~m}}}-\frac{\pi l w(1-r)}{\left\langle\lambda_{\mathrm{c}}\right\rangle}
$$

which means that $N_{3, \mathrm{~m}}$ can be determinated directly from the $A_{\mathrm{m}}(n, l)$ values. Omittance of the spectral term in Eq. (3.10) produces errors of a few percent in the value calculated for the particle size.

Finally, because determinations of particle size and strain can be performed independently of distortion [Eq. (3.9)] and particle size [Eq. (3.8)] in the standard specimen, it might be suggested that, for example with a view to extinction problems, for the determination of particle size a slightly deformed standard specimen should be used and for the determination of distortion colloids as a standard specimen should be used.

\section{Acknowledgements}

We are grateful to Ir. R. Delhez for stimulating discussions and for supplying the Si (444) profile. Professor B. Okkerse and Dr. F. W. Schapink critically read the manuscript.

Financial support of the Stichting voor Fundamenteel Onderzoek der Materie (F.O.M.) is gratefully acknowledged.

[5] E. O. Brigham, The Fast Fourier Transform, Prentice Hall, Englewood Cliffs, New Jersey 1974, pp. $91 \mathrm{ff}$.

[6] R. A. Young, R. J. Gerdes, and A. J. C. Wilson, Acta Cryst. 22, 15 (1966).

[7] A. J. C. Wilson, Acta Cryst. 23, 888 (1967).

[8] A. J. C. Wilson, Acta Cryst. A 24, 478 (1968).

[9] A. J. C. Wilson, Acta Cryst. A 25, 584 (1969). 
[10] T. P. O'Holleran, H. A. McKinstry, and V. S. Stubican, Phil. Mag. 35, 485 (1977).

[11] N. C. Halder and E. E. Johnston, Z. Naturforsch. 30 a, 825 (1975).

[12] J. P. Urban, J. Appl. Cryst. 8, 459 (1975).

[13] T. C. Huang and W. Parrish, Appl. Phys. Letters 27, 123 (1975).

[14] Assuming that a high angle reflection of a standard specimen is broadened only by the X-ray spectrum used [13], a high angle reflection has to be measured again with change of X-ray spectrum.

[15] R. Delhez, Th. H. de Keijser, and E. J. Mittemeijer. J. Phys. E: Sci. Instrum., in the press.

[16] R. Delhez and E. J. Mittemeijer, J. Appl. Cryst. 8, 609 (1975).

[17] A. H. Compton and S. K. Allison, X-rays in Theory and Experiment, MacMillan, London 1935.
[18] All $(h k l)$ reflections can be considered as (00l) ones: A. R. Stokes and A. J. C. Wilson, Proc. Cambridge Philos. Soc. 40, 147 (1944) ; B. E. Warren, Acta Cryst. 8, 483 (1955).

[19] R. Delhez and E. J. Mittemeijer, J. Appl. Cryst. 9, 233 (1976).

[20] For an ideal standard specimen $A_{0} \mathrm{~S}(n)=1$ [infinite particle size] for all $n$. In practice the standard specimen has a large, however finite, particle size $N_{3,0}$. Hence $A_{0} \mathrm{~S}(n)$ tends to zero at large $n$. Combined with the effect of counting statistics this may be responsible [c.f. Eq. (3.7)] for the large spurious ripples in the Fourier coefficients of a deconvoluted line profile at large $n\left(\mathrm{n}>N_{3,0}\right)$.

[21] M. A. Zemitis, A. Kidron, and J. B. Cohen, Scripta Met. 6, 875 (1972).

[22] Th. H. de Keijser and E. J. Mittemeijer, Phil. Mag. 36, 1261 (1977). 